\title{
UNUSUAL CASE OF DISSEMINATED SARCOMA IN A GHILD
}

BY

\author{
ERIC PRITCHARD, M.D., F.R.C.P., \\ Medical Director of the Infants Hospital, London, \\ AND \\ DAVID H. HALER, M.B., B.S., \\ Pathologist to the Infants Hospital, London.
}

A remarkable case of malignant disease in a young child, apparently arising from a growth in the caecum, was recently under our care. The diagnosis during life presented great difficulty and was finally made on the pathological findings at post mortem.

\section{Case report.}

The child, at the age of six months, was first brought to the Infants Hospital for treatment for sleeplessness and continuous crying, symptoms which were at the time regarded as due to teething, and treated as such. From that time forward until admission to the hospital on September 1st, 1933, the child attended intermittently at the out-patient department of the hospital for a variety of symptoms-colds, colitis, intestinal disturbances, throat troubles, and other minor ailments. The tonsils were removed by Mr. P. Franklin in August, 1931, when the child was two years of age. On the 29th August, 1933, at the age of four years, he was again brought to the hospital for diarrhoea, threadworms, and a rash on shoulders and arms. On this occasion he was seen by one of the clinical assistants, but no note was made of the size of the liver, although there is mention that the abdomen was enlarged. On September 1st, 1933, the child was again brought to the hospital with a note from Dr. S. E. Knowles, of New Cross, calling attention to the size of the abdomen and the difficulty in breathing. He suggested that the case might be one of tuberculosis. The child was then admitted to the hospital as an in-patient.

On examination it was found that the liver was enormously enlarged, extending almost to the umbilicus. It was uniformly smooth and not hard. Nothing else could be discovered in the abdomen to account for the general enlargement. There was no free fluid, no tumour, and no obvious distension of the intestines. The child's breathing was rapid and laboured, and on the second day after admission the temperature rose, together with the respiration and pulse rate. During the fourteen days which intervened between the date of admission and the child's death, the general condition rapidly grew worse, with general rise in temperature, pulse rate, and respirations. There was a very profuse perspiration, and a rash broke out on the face, which at first was regarded as sordes, but later on as an unusual form of tuberculide.

The physical signs of the chest were somewhat indefinite, and hardly sufficient to account for the dyspnoea and respiratory distress, but clinical evidence of double pleuritic effusion with collapse of lung at both bases gradually developed, and was confirmed on September 4th by X-ray examination. 
Although the symptoms and general condition of the child on the whole favoured a diagnosis of tuberculosis, the condition of the liver and the rapid down-hill course of the child left us in considerable doubt as to whether there was not some other interpretation.

The urinary report threw little light on the case, but acetone and a trace of diacetic acid and urates were found to be present. A blood examination showed a high degree of acidosis. The throat and nasal swabs gave negative results for K.L.B. The differential blood count was as follows:-erythrocytes 5,375,000, haemoglobin 112 per cent., colour index 1.04, leucocytes 7,200.

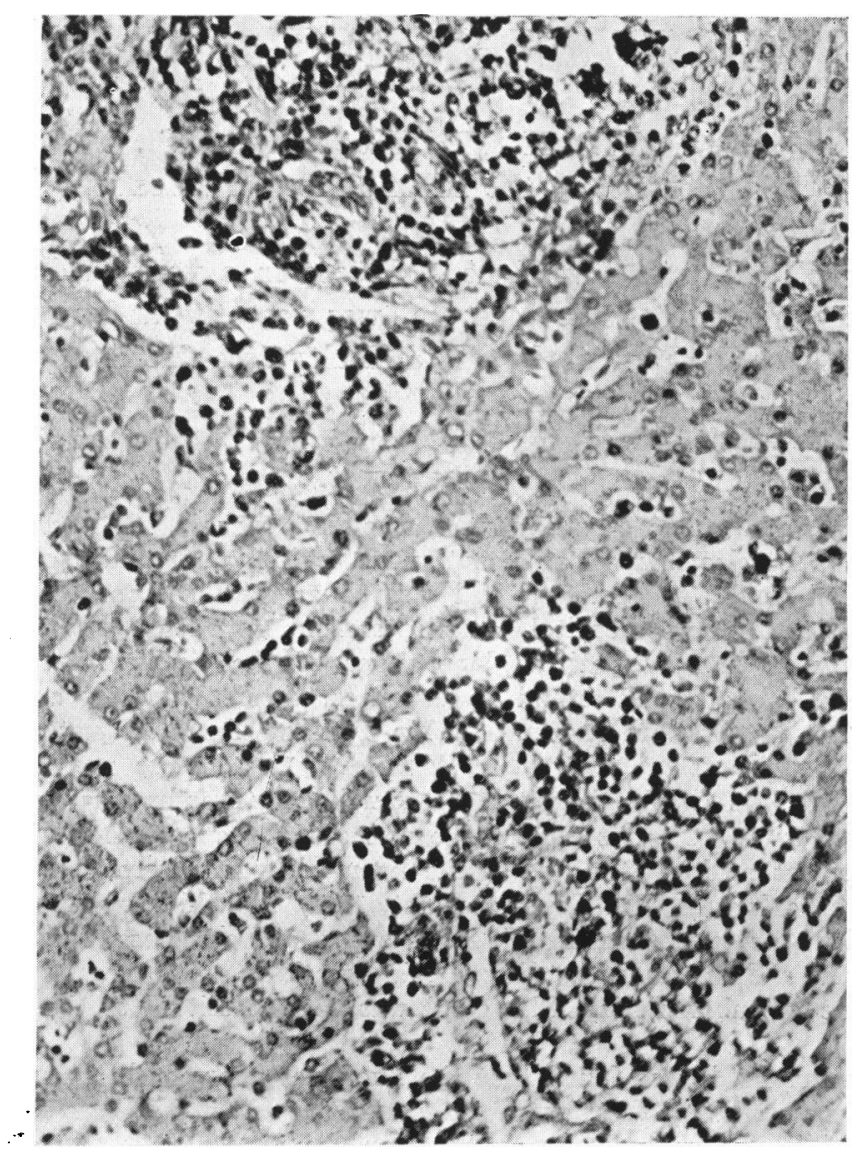

FIG. 1.-Section of liver showing the neoplastic invasion.

Differential count:- polymorphs $47 \cdot 2$ per cent., eosinophils $2 \cdot 4$ per cent., neutrophil myelocytes 10.4 per cent., basophils 0.4 per cent., large hyaline cells 1.2 per cent., lymphocytes 37.2 per cent. One normoblast seen in counting 250 cells-i.e., 29 per c.mm., erythrocytes otherwise normal. Mean diameter of erythrocytes (Eve's method) 7.30 microns. No tubercle bacilli were discovered in faeces, or urine, after the employment of both direct and concentration methods. The skin reaction was positive to human, but negative to bovine tuberculin.

On the 13th day after admission, the dyspnoea becoming worse, the right side of the chest was aspirated, and 5 c.c. of blood stained fluid was withdrawn. In this there 
was a rather heavy coagulum, with a considerable number of white cells, mainly lymphocytes; no organisms could be discovered by direct examination, and only one colony grew on culture. This was found to be staphylococcus aureus, and was probably a contamination. The fluid was inoculated into a guinea-pig with negative result.

From this time forward, the child went rapidly down-hill, with delirium at times, but with no definite meningeal symptoms.

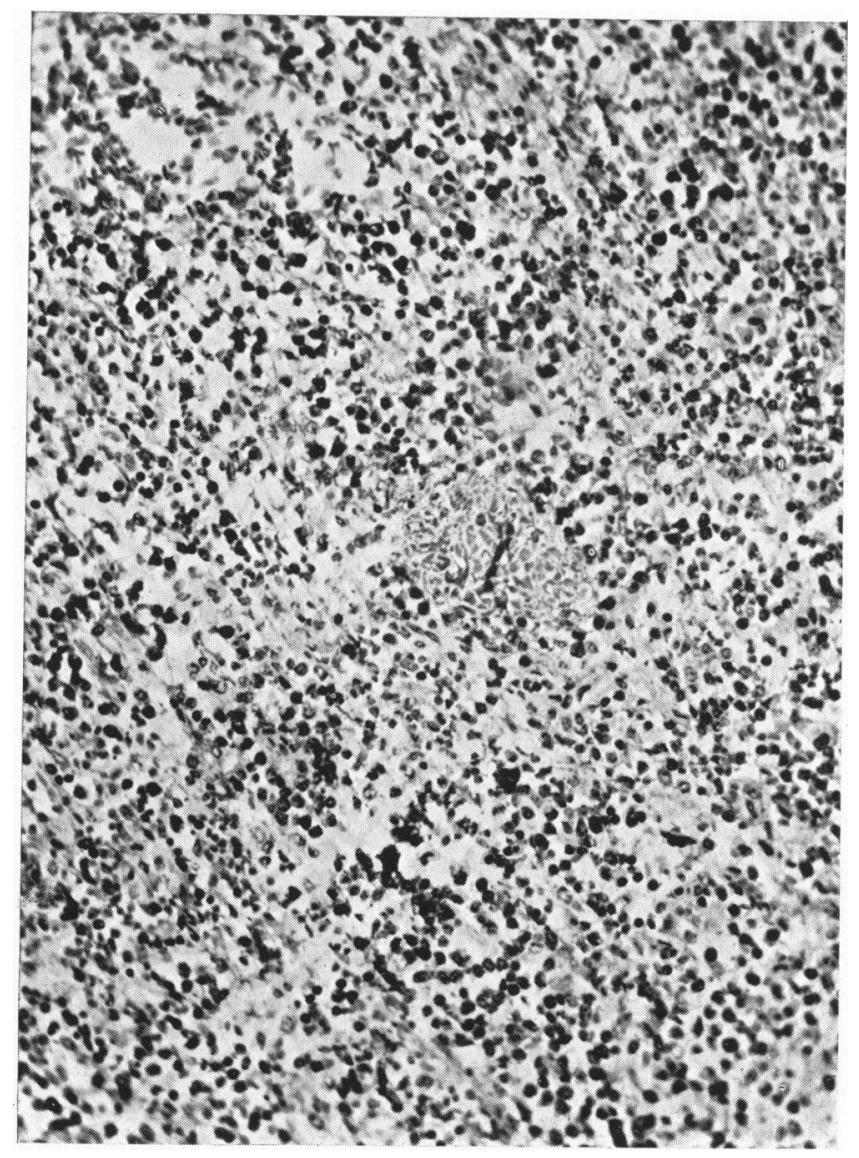

Fig. 2.-Section of spleen, showing great destruction of normal architecture.

\section{Discussion.}

The clinical interest in this case lay chiefly in the difficulty of making a definite diagnosis during life. There was some evidence that the case might be one of tuberculosis, and yet the general clinical picture was not entirely in accordance with this view. The uniform and extensive enlargement of the liver was not altogether suggestive of either tuberculosis or of a neoplasm, and with the exception of a few pedunculated excrescences on the 
skin, we could find no evidence of primary or secondary growth. We had intended to excise one of these and submit it to microscopical examination, but the child died before this investigation could be carried out.

Although the post-mortem pathological report which follows shows clearly that this case was one of a very widely disseminated malignant

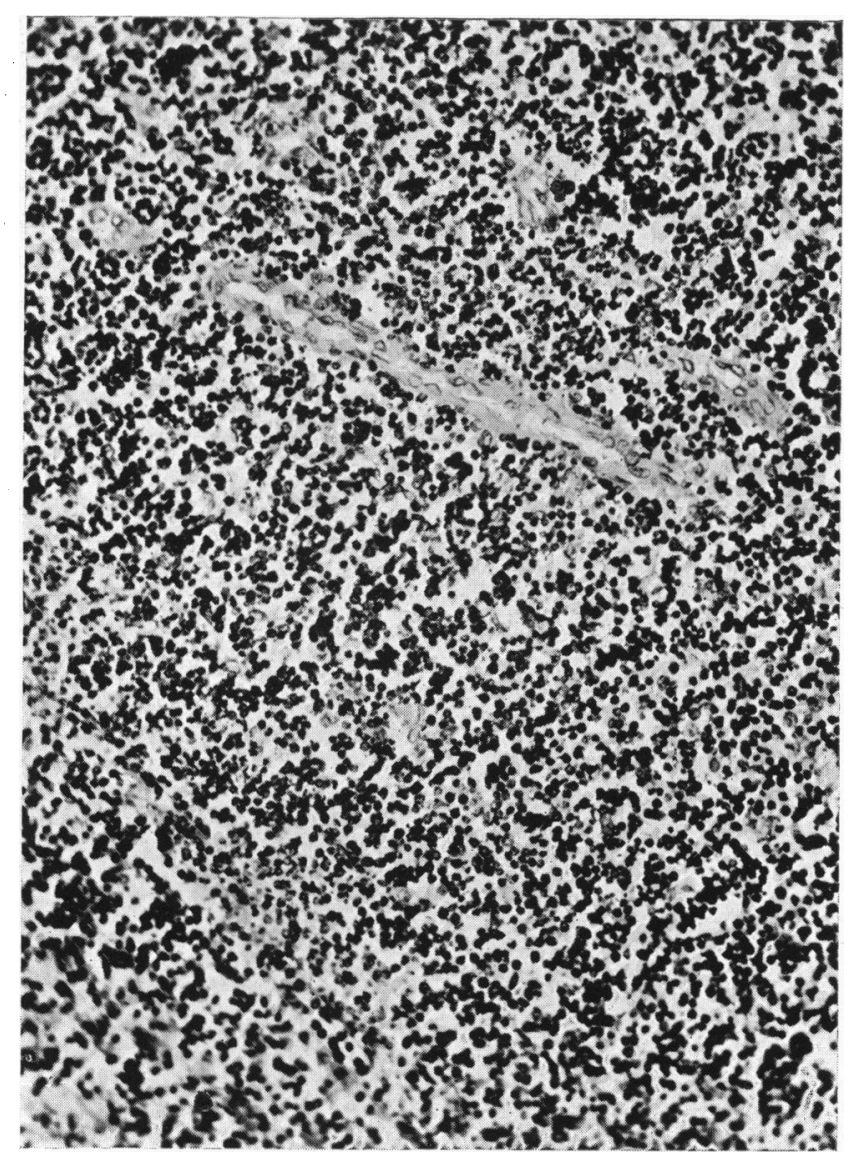

FIG. 3.-Section of tumour of the caecum (the possible primary lesion).

growth, with the exception of the pedunculated excrescences on the skin referred to above and the somewhat unusual differential blood count, there was little evidence to justify a diagnosis of malignant disease. It is interesting to note that throughout the case there runs a connected history of bowel disturbances, which inclines us to the belief that the primary growth may possibly have been in the caecum. The co-existence of tuberculosis is probably an unimportant event in the history. 


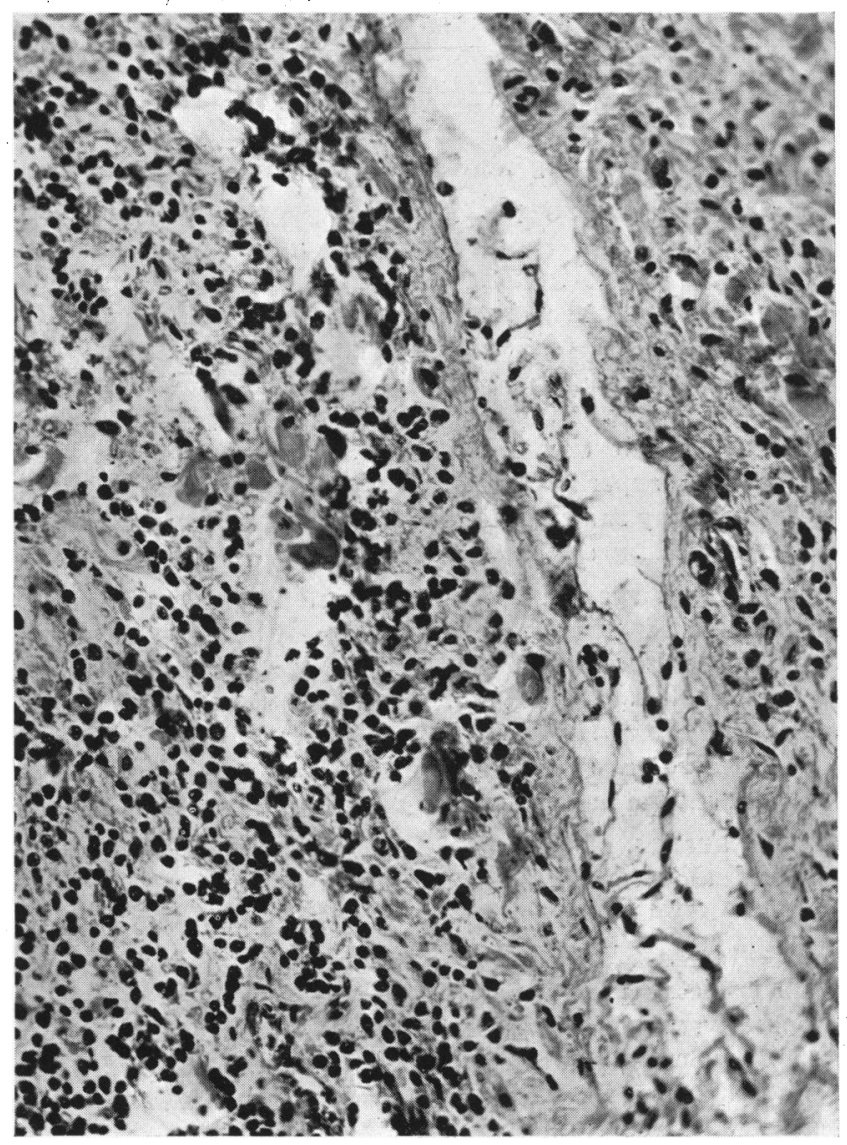

FIG: 4,-Section of gall bladder, showing destruction of normal tissue.

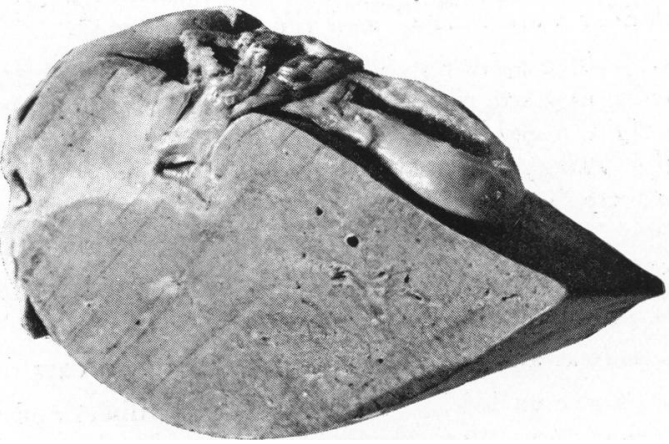

Fig. 5.-Macroscopical view of liver with gall bladder in situ. 


\section{Pathological report (post mortem).}

Very wasted malc, 'ild with excessive labial sordes. Many small reddish pedunculated lesions of skin present. Abdomen markedly distended. Rigor mortis passing off. Post-mortern staining well seen.

Almentary system. Mouth, pharynx, oesophagus, stomach, duodenum, and jejunum, normal. The ileum showed marked hypertrophy of Peyer's patches. Minute intramural tumour-like lesions were present along the longitudinal axis of the caecum with some superficial ulceration. The large intestine was distended. The appendix was thickened, directed upwards and adherent to the right suprarenal capsule. A large white ' cauliflower' tumour (see illustrations), occupied the whole of the posterio-inferior aspect of the caecum, but did not obstruct the opening of the ileo-caecal valve. The whole of the bowel in this region was studded with minute neoplastic growths with superficial ulceration. The mesenteric lymph nodes were large, hard, discrete, mostly showing definite haemorrhages on section. The intestines contained much foul-smelling and altered blood.

THE LIVER was very large, weighing $1,210 \mathrm{gm}$. (normal for age, $500 \mathrm{gm}$.). The edges were rounded and the capsule thickened, with numerous subcapsular deposits varying in size from a hemp seed to a lentil. On section, the liver was studded with innumerable white infiltrations in the interlobular regions. The gall bladder deserves special comment. Before opening it appeared completely solid, and on section was found to have greatly thickened walls, and to be devoid of bile, but containing some clear mucus in the fundus. The bile ducts were normal and patent.

Cardiovascular system. The pericardium was normal, with no effusion. The heart was small and the valves and great vessels normal. The muscle was friable and pale.

Respiratory system. The larynx was normal. There was some muco-pus in trachea. The parietal pleura was beset with numerous flat white nodules running in intercostal grooves, more marked on the right side. The right pleural cavity contained 600 c.c. and the left 250 c.c. of blood stained serous fluid with fine coagula. The diaphragmatic pleura was beset with nodules similar to above, but the visceral pleura was free from nodules. The right lung collapsed. One hilar lymph node was enlarged to size of walnut, caseous and blocking the bronchus, but showing no ulceration. Tubercle bacilli were demonstrable in stained sections and direct films. The left lung was only partially collapsed. No caseation of hilar nodes present. No deposits found in or on lung surfaces. The mediastinal lymph nodes were greatly enlarged, hard, discrete, mottled and firm on section.

Genito-urinary system. Both kidneys were fatty, pale, and studded throughout their substance with multiple miliary circumscribed haemorrhagic and necrotic areas. The ureters and bladder were normal and the testes normal.

Ductless glasps. The spleen weighed $350 \mathrm{gm}$. and was large, hard and studded throughout with white deposits similar to those in liver. The left suprarenal capsule was normal. The right appeared large and involved in a mass of whitish material anchoring it to the kidney, appendix, and right side of diaphragm. One small circumscried and partly haemorrhagic nodule was found in the usual site at apex of capsule. The thymus, thyroid, pituitary, and pineal glands were apparently normal.

Lymphatic glands. The axillary, inguinal, and cervical glands were all hard, discrete and grey-white in colour.

THE SKULL was normal and the brain was normal. The ears did not contain pus.

SKin TUmours. These varied in size from lentil to filbert and were pedunculated, red-purple, and showed early ulceration. They were distributed in a linear fashion on the face and back, particularly over sacral region. 
Histological investigation.-Sections were taken of a skin tumour, the liver, spleen, kidney, tumour of caecum, right suprarenal, mass from coeliac axis, gall bladder, lymph node from coeliac region, hilar lymph node (caseous), and a mesenteric lymph node.

All these sections were stained with haematoxylin and eosin, and, in addition, were examined by Schmorl's method for acid fast bacilli and by Gram's method for micro-organisms. Acid fast bacilli were found only in sections from the hilar lymph node, Gram positive cocci were seen in the skin tumour only. The remaining sections showed various degrees of destruction of normal tissue and replacement by neoplastic cell growth. The invading tissue was of fine alveolar structure and composed mainly of small round cells with a deeply staining cytoplasm, the nuclei of which showed varying degrees of nuclear mitosis. There were also large blood spaces of irregular type and there was no attempt at reproduction of tissue form. In other words, the condition was one of a widely disseminated invasive roundcelled sarcoma. With regard to the primary tumour, it is possible, but not conclusively demonstrable, that the original growth was in the caecum, and that the condition present was a wide dissemination from this primary focus. On the other hand, from the abnormal blood picture, the generalized enlargement of the lymphatic glands, and wide distribution of the lesions, the inference is that we were here dealing with an abnormal border-line case between sarcomatosis of a diffuse type and the aleukaemic stage of a myelogenous leukaemia.

The investigations performed during life were carried out as routine investigations and the findings were considered to point to an unusual reaction to a generalizing tuberculosis. There was at no time any clinical indication that the post-mortem findings would take the above described form.

At the autopsy itself we were still left with the choice of several alternative diagnoses, and of these the following seemed the more probable:-

(a) Generalized tuberculosis of the hypertrophic granulomatous type.

(b) An atypical Hodgkin's disease.

(c) Malignant disease of hypernephromatous or sarcomatous type.

The actual diagnosis rests on the histological examinations subsequently carried out, some of the more typical of which are illustrated by microphotographs (Fig. 1 to 4 : magnified 360 diameters) taken by Mr. J. G. Bradbury, F.R.M.S., F.R.P.S.

From the post-mortem findings it will be seen that there was some justification for our failure to arrive at a correct diagnosis during life. 